\title{
An instrument to obtain the correct biaxial hyperelastic parameters of silicones for accurate DEA modeling
}

Samuel Rosset, Luc Maffli, Simon Houis, Herbert. R. Shea

Proc. SPIE 9056, Electroactive Polymer Actuators and Devices (EAPAD) 2014, 90560M (March 8, 2014); doi:10.1117/12.2044777

Copyright 2014 Society of Photo-Optical Instrumentation Engineers. One print or electronic copy may be made for personal use only. Systematic electronic or print reproduction and distribution, duplication of any material in this paper for a fee or for commercial purposes, or modification of the content of the paper are prohibited. 


\title{
An instrument to obtain the correct biaxial hyperelastic parameters of silicones for accurate DEA modelling
}

\author{
Samuel Rosset, Luc Maffli, Simon Houis and Herbert R. Shea \\ Ecole polytechnique fédérale de Lausanne, Switzerland
}

\begin{abstract}
The analytical formulas describing the behaviour of dielectric elastomer actuators (DEAs) are based on hyperelastic strain energy density functions. The analytical modelling of a DEA will only lead to meaningful results if the dielectric elastomer can be accurately represented by the chosen hyperelastic model and if its parameters are carefully matched to the elastomer. In the case of silicone elastomers, we show that the strain energy density of a thin elastomeric membrane depends on the maximum deformation the membrane was previously submitted to (Mullins effect). We also show that using model parameters coming from an uniaxial pull-test to predict the behaviour of the elastomer in an equi-biaxial configuration leads to erroneous results. We have therefore built a measurement setup, which allows testing thin elastomeric membranes under equi-biaxial stress by inflating them with a pressure source. When modelling a DEA under equi-biaxial stretch, the measurement data can be used directly, without the need of an hyperelastic model, leading to voltage-stretch prediction closer the the measured stress-stretch behaviour of the dielectric membrane.
\end{abstract}

Keywords: Hyperelastic models, Mullins effect, silicone, dielectric elastomer actuators

\section{INTRODUCTION}

The modeling of dielectric elastomer actuators (DEAs) voltage-stretch behaviour formalized by Suo, ${ }^{1}$ and used in many theoretical analyses of such actuators ${ }^{2-5}$ is based on using an hyperelastic material model to describe the strain energy density of the dielectric elastomeric membrane. Among the different existing hyperelastic models, the Gent model is often used in theoretical analyses of DEAs, ${ }^{3-8}$ because it can model the limiting stretch that an elastomer can sustain, when its chains are completely stretched. Some other common models, such as neo-Hookean or Yeoh, do not show the existance of a maximal stretch. The Gent free energy density is given by $:^{9}$

$$
w=-\frac{\mu J_{m}}{2} \ln \left(1-\frac{I_{1}-3}{J_{m}}\right),
$$

where the two parameters of the model $\mu$ and $J_{m}$ are respectively the shear modulus, and a parameter defining the stretch at which the stress diverges.

If the model parameters ( $\mu$ and $J_{m}$ for Gent) are known for a material, then its stress state can be calculated for any arbitrary stretching. The value of the parameters are often determined using a uniaxial pull test on a long an narrow sample, fitting the experimental stress-stretch data points to the chosen model. However, in many cases the dielectric membrane in a DEA is in a biaxial stress state, which can even be equi-biaxial in some particular cases such as the typical test actuators depicted on figure 1, which consists of an equi-biaxiallyprestretched membrane at the center of which a small (compared to the total membrane area) circular active zone is defined. Although the parameters obtained in a particular stretching condition (such as uniaxial) should be valid for any other configuration (such as equi-biaxial), this is only true if the chosen hyperelastic strain energy density function is an accurate model of the elastomer. Even if the model provides a good fit for the experimental data in one stretching condition, it is only a good representation of the strain energy of the tested material, if it can also model it accurately in other stretching conditions.

In order to verify the applicability of the Gent model to the silicone elastomers that we use to make actuators, we have experimentally tested thin silicone membranes in two different stretching conditions: a conventional

contact author: samuel.rosset@a3.epfl.ch

Electroactive Polymer Actuators and Devices (EAPAD) 2014, edited by Yoseph Bar-Cohen, Proc. of SPIE Vol. 9056, 90560M · @ 2014 SPIE · CCC code: 0277-786X/14/\$18 · doi: 10.1117/12.2044777

Proc. of SPIE Vol. 9056 90560M-1 

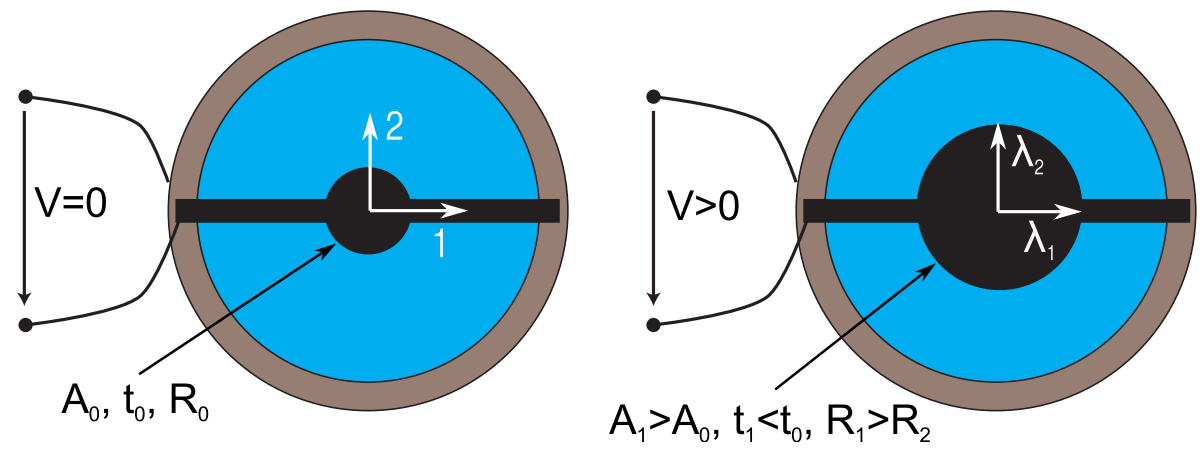

Figure 1. Circular actuator on an equi-biaxially prestretched membrane. The application of a voltage causes the electrode to expand in plane. The stress state of the dielectric in the active region remains equi-biaxial.

a)

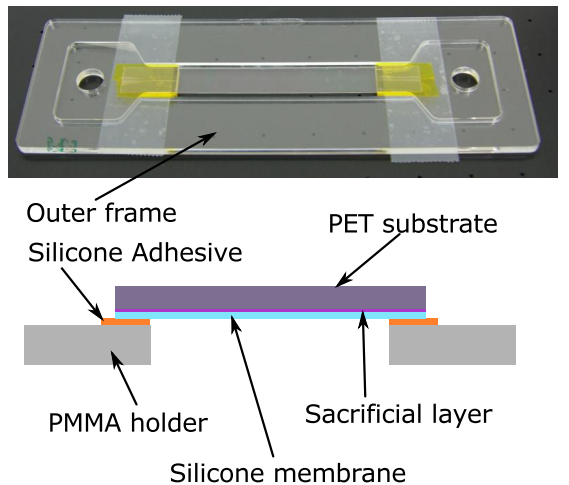

b)

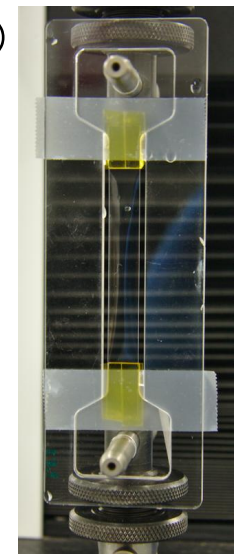

c)

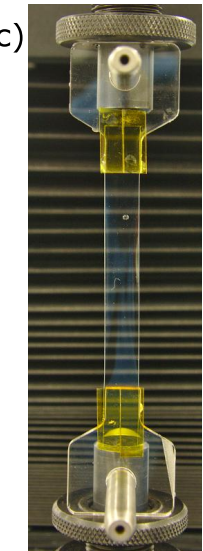

Figure 2. Sample preparation for uniaxial pull-test. a) A thin $10 \times 90 \mathrm{~mm}^{2}$ strip of PET/sacrificial layer/silicone sandwich is fixed on two PMMA holders with double-sided silicone tape. The two holders are held in a rectangular frame in order to prevent any motion. b) After removal of the PET substrate, the sample is mounted on the pull-tester. The frame around the holders ensures that the sample is not strained while fixing it on the machine. c) The frame is removed, leaving the sample fixed to the two holders and ready to be stretched.

uniaxial pull-test and an equi-biaxial stretching test using the inflation of a bubble (see section 3). In this paper we show that different parameters for the Gent model are obtained from uniaxial and equi-biaxial tests. In addition, we show that because of the existence of a stretch-induced softening of the silicones, also known as Mullins effect, ${ }^{10}$ the strain energy function does not only depend on the stretch state of the material, but also on the maximal stretch the sample has seen before. This stretch history dependence of the mechanical properties of silicones makes modelling particularly tricky. Indeed the mechanical properties of the dielectric elastomer membranes depend on many parameters not always easy to control, for example if the membrane is deformed during fabrication (when peeled off from the substrate on which it has been casted).

\section{UNI-AXIAL PULL TEST}

One of the most straightforward way to fit experimental data to an hyperelastic model in order to identify the value of its parameters is to do an uniaxial pull-test. From the force versus displacement values, the stress versus stretch can be easily calculated and fitted to the theoretical stress-stretch law derived from a given strain energy function. In the case of the Gent model, and due to the existence of the stress divergence for a finite stretch value, one might be tempted to stretch the sample until it breaks in order to have a good approximation of the $J_{m}$ parameter. Such a one shot measurement unfortunately leads to wrong parameters values in the case of silicones. This is due to the Mullins effect, which is a softening of filled rubber dependent on the largest strain the material has previously seen. ${ }^{10}$

To show the importance of this effect, we have conducted uni-axial pull-tests on the silicone CF19-2186 by Nusil (CF19), which is one of the silicones we commonly use in our lab to make DEAs. In order to get results 

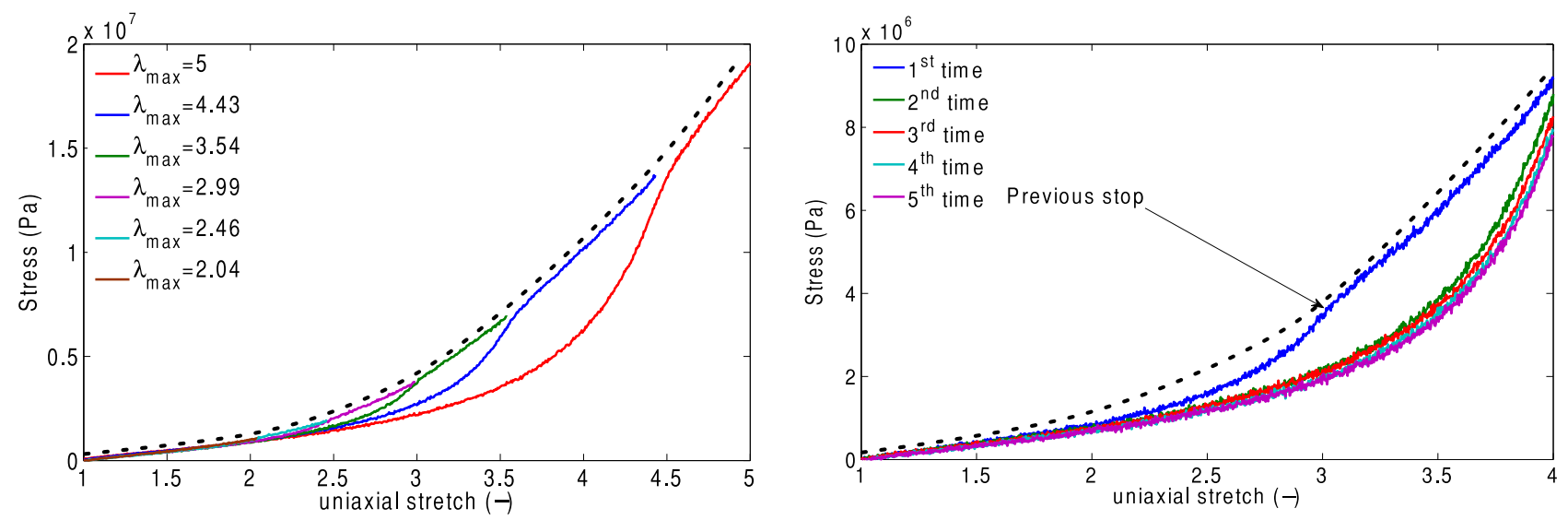

Figure 3. Left: repeated uniaxial pull-tests on a CF19 sample with increasing maximal stretch clearly show the softening of the material. Right: repeated uni-axial test up to a stretch ratio of 4 on a Sylgard 186 sample shows that the softening effects happens mostly after the first stretching. The subsequent tests exhibit a limited further softening.

as meaningful as possible for our DEAs, we have used the exact same fabrication process to produce the pulltest samples that we use for our DEA membranes. Indeed our experience has shown that a silicone sample's mechanical properties are dependent on its fabrication parameters (thickness, curing temperature, presence of solvent, etc.). In order to show the presence of a Mullins effect, we also want to make sure that the thin silicone sample is not subjected to any deformation during fabrication and installation on the pull-test setup: the silicone is blade-casted to a height of $58.3 \mu \mathrm{m}$ on a A4 PET foil (Melinex ST-506 from DuPont Teijin films) previously coated with a water soluble sacrificial layer. After cross-linking at $80^{\circ}$ in an oven, the PET/sacrificial layer/CF19 sandwich is laser-cut into strips of $10 \times 90 \mathrm{~mm}^{2}$. A sample is fixed with double-sided silicone adhesive to two holders which are maintained in place in an outer frame (figure $2 \mathrm{a}$ ). The adhesive force provided by the tape is enough to hold the sample even at large stretch. The outer frame holds all of the part together and prevents them from moving relative to each other. The assembly is then dipped into a hot water bath to dissolve the sacrificial layer. This allows to remove the PET substrate on which the silicone was casted without deforming the thin silicone sample. Then the assembly is mounted on the pull-tester (figure $2 \mathrm{~b}$ ). There are holes in the two holders which match pins on the machine. Because the two holders are locked in the outer frame, the silicone sample is not strained during its installation on the setup. Finally, the outer frame is removed and the sample is ready to be tested (figure $2 \mathrm{c}$ ). The sample has not been strained either during the release from the casting substrate or during installation on the testing equipment, which is important if one wants to obtain meaningful data.

The sample was then tested up to increasing maximal stretches of 2.04, 2.46, 2.99, 3.54, 4.43, and 5 (figure 3 left). The maximal stretch of each segment was chosen to compare with bi-axial measurements which will be presented later (c.f. section 3). It can be seen that for a stretch above 2, the stress-stretch curve is different depending if the sample is deformed for the first time at this level, or if it has already seen such stretches previously. In particular, the slope of the curve changes when the sample reaches a larger deformation compared to what it has seen during the preceding test. The black dashed line represents the curve that would have been obtained by stretching the sample up to $\lambda=5$ in one single step. It is clear that using this curve to fit an hyperlastic model would lead to erroneous predictions of the sample's stress-stretch behaviour. This softening has also been observed on DowCorning Sylgard 186, another silicone that is used in our lab to make DEAs (figure 3 right). In that case, a sample prepared in the same manner than for the CF19 test was stretched 5 times up to $\lambda=4$ after having been previously stretched to $\lambda=3$. During the first test, the change of behaviour once the sample is entering a stretch range it didn't see before $(\lambda>3)$ can clearly be seen by an abrupt change of slope. The second test looks very different, but the subsequent tests (3 to 5 ) lead to results which are similar to the second test. The black dashed line represents the stress-stretch curve that would have been obtained if a pristine sample had been stretched to $\lambda=4$ a single time. It is clear that fitting an hyperelastic model on this curve would not accurately model the behaviour of the sample stretched to $\lambda=4$, which would now follow the curve of test 2 to 5 , if it were stretched again to the same value. 


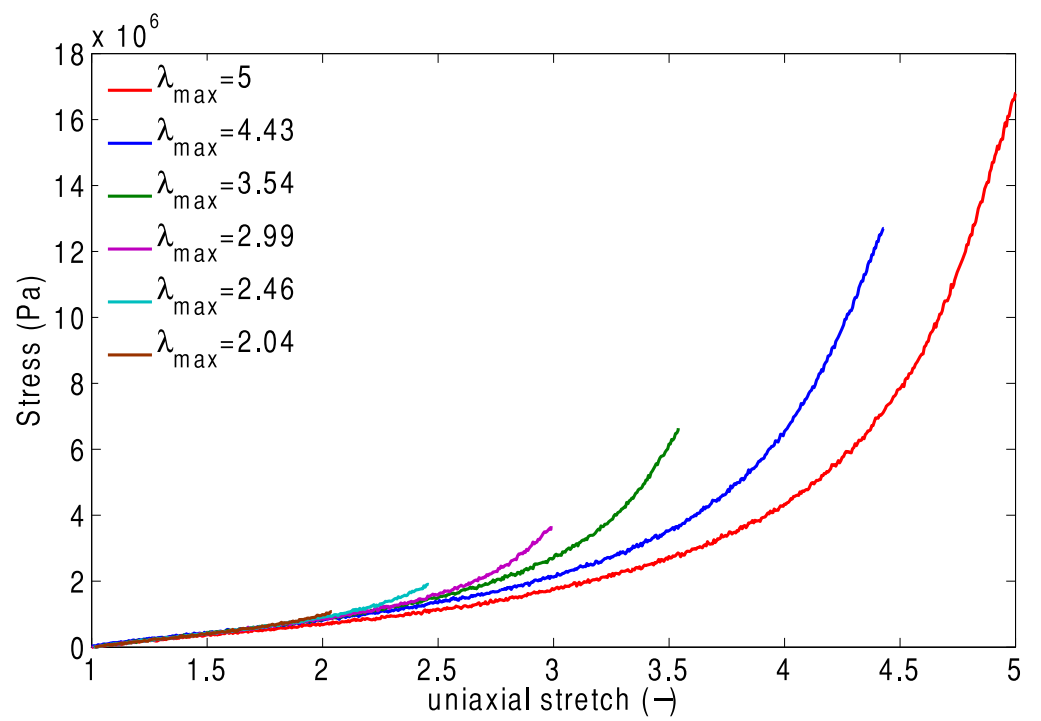

Figure 4. Stress-stretch curve on the CF19 silicone sample obtained for different values of the maximal stretch value. The sample was stretched 3 times to each maximal stretch, and the last measurement is reported on the graph.

\begin{tabular}{|l|c|c|c|c|c|c|}
\hline Maximal stretch & $\mathbf{2 . 0 4}$ & $\mathbf{2 . 4 6}$ & $\mathbf{2 . 9 9}$ & $\mathbf{3 . 5 4}$ & $\mathbf{4 . 4 3}$ & $\mathbf{5}$ \\
\hline$\mu(\mathrm{MPa})$ & 0.243 & 0.247 & 0.195 & 0.186 & 0.174 & 0.149 \\
\hline$J_{m}$ & 17.02 & 12.86 & 12.75 & 15.65 & 23.05 & 28.43 \\
\hline
\end{tabular}

Table 1. Values of the shear modulus $\mu$ and the $J_{m}$ parameter obtained by fitting the Gent model to the curves of figure 4.

The observed softening effect has the following consequences relative to applying hyperelastic models to pull-test data:

- The fitting should not be performed on the first measurement, but the sample must be stretched at least twice to the same value. Figure 4 shows the stress-stretch behaviour of CF19 obtained after the 3rd stretching to each maximal value. This constraint prevents stretching a sample until it ruptures in one single measurement.

- One shouldn't use an hyperelastic model to extrapolate the stress-stretch behaviour past the maximal tested stretch, as the mechanical behaviour of the sample will be different past this point.

- The material cannot be characterized by a single set of model parameters, as its mechanical properties do not only depend on the material, but also on its stretching history. Table 1 gives the Gent parameters $\mu$ and the $J_{m}$ of Nusil CF19 for different values of $\lambda_{\max }$, as obtained by fitting on the uniaxial pull-test data of figure 4. It can be noted that with increasing maximal stretch, the material becomes softer (the shear Modulus decreases).

- One alternative would be to use a material model including the stretch-induced softening.

In theory, if one assumes that a material behaves according to a given hyperelastic model (such as the Gent model), then once we have obtained its parameters via a uniaxial pull-test, we can calculate its stressstretch behaviour for a different stretching case, such as equi-biaxial (to take the example of the simple actuator presented in section 1). Because the model parameters are valid only for a limited stretching range and cannot be extrapolated beyond the maximal stretch applied to the sample, we need to define the biaxial stretching range equivalent to the tested uniaxial range in which a given set of parameters is valid. We take the strain energy density as the reference quantity: if the sample was uniaxially stretched to a maximum of $\lambda_{u}$, then the sample has seen an energy density between 0 and $w_{\max }$, and the maximum equi- biaxial stretch $\lambda_{b}$ that can be applied to the material in order to remain in the same conditions than for the uni-axial test is the stretch that 


\begin{tabular}{|c|c|c|c|c|c|}
\hline $\mathbf{I}_{\mathbf{1}}$ & $\mathbf{5 . 1 5}$ & $\mathbf{6 . 8 6}$ & $\mathbf{9 . 6 3}$ & $\mathbf{1 3 . 1}$ & $\mathbf{2 0 . 1}$ \\
\hline$\lambda_{u}$ & 2.04 & 2.46 & 2.99 & 3.54 & 4.43 \\
\hline$\lambda_{b}$ & 1.58 & 1.84 & 2.19 & 2.56 & 3.17 \\
\hline
\end{tabular}

Table 2. Equivalence between the uniaxial stretch $\lambda_{u}$ and the equibiaxial stretch $\lambda_{b}$ for different values of $I_{1}$. The values are chosen to match the maximal stretches used for the uniaxial test.

will lead to the same energy density $w_{\max }$. From (1) we deduce that the first invariant must therefore be equal in the uniaxial case and the equi-biaxial case (or any other arbitrary stretch configuration), i.e $I_{1, u}=I_{1, b}$, the subscripts $u$ and $b$ referring respectively to the uniaxial case and equi-biaxial case. Table 2 shows the equivalence between the uniaxial stretch $\lambda_{u}$ and the equibiaxial stretch $\lambda_{b}$ for different values of $I_{1}$. The values are chosen to match the maximal stretches used for the uniaxial test of the CF19 sample shown above. For example, we assume that the Gent parameters $\mu=0.243 \mathrm{MPa}$ and $J_{m}=17.02$, which are valid for samples which have seen an uniaxial stretch up to 2.04, are also valid to model the same material in equi-biaxial extension for a stretch value up to 1.58 .

However, even with the equi-biaxial stretch range equivalence explained above, the question of the validity of the model parameters obtained from an uniaxial pull-test to model an equi-biaxial case remains open. It is for example possible that the extent of the softening effect is quite different between the two situations, the polymer chains being pulled along one direction in the equi-biaxial case, and in two perpendicular directions for the equi-biaxial case. Because the actuator we want to model is in an equi-biaxial state, it would be more accurate to fit the hyperelastic model on equi-biaxial stress-stretch data. The next section presents a test setup designed to acquire this information from thin silicone membranes, and the results are compared with the model parameters obtained from the uniaxial pull-test

\section{BIAXIAL TEST}

Placing a thin membrane in a perfectly equi-biaxial stress state is not straightforward. One of the possibilities consists in inflating a suspended membrane by a compressed gas, as proposed by Reuge et al. ${ }^{11}$ Because of the fixed boundary conditions at the periphery of the membrane, the entire surface of an inflated membrane is under a complex location-dependent stress state. However, the apex of the inflated dome can be considered to be under an equi-biaxial stress state, and in this region the hoop equi-biaxial stress in the membrane $\sigma_{b}$ is linked to the applied pressure difference $\Delta p$ by the following equation: ${ }^{11}$

$$
\sigma_{b}=\frac{\Delta p R}{2 t}
$$

where $R$ is the radius of curvature of the inflated membrane at the apex and $t$ the thickness of the membrane in the same region, which can be obtained from the initial thickness $t_{0}$ using the volume invariance: $t=t_{0} / \lambda_{b}^{2}$, with $\lambda_{b}$ the equibiaxial stretch at the membrane center, thus leading to:

$$
\sigma_{b}=\frac{\Delta p R \lambda_{b}^{2}}{2 t_{0}}
$$

In order to extract the equi-biaxial stress-stretch characteristic of a membrane, it is therefore necessary to inflate the membrane with a known pressure and to measure the stretch and curvature radius at its center. Because the total membrane cannot be considered to be under an equi-biaxial stress state, the key is to measure these two parameters at the center of the membrane only. Reuge et al. have patterned a white silicone paste rectangle at the center of their $2 \mathrm{~mm}$-thick membranes in order to identify the region of interest. Because we are working with much thinner membranes (in the $50 \mu \mathrm{m}$ range) using a similar approach would have too much impact on the mechanical properties of the membrane. Instead, we patterned two thin parallel lines near the center of the membrane with the carbon black/silicone mixture that we use for the electrodes of our DEAs. These two black lines are easily identified on the otherwise transparent membrane.

Our test setup (figure 5) consists of a membrane holder which can be pressurised by a regulated microfluidic flow control system from Fluigent. A $5 \mathrm{Mpx}$ camera is used to take pictures of the inflated membrane from 
a)
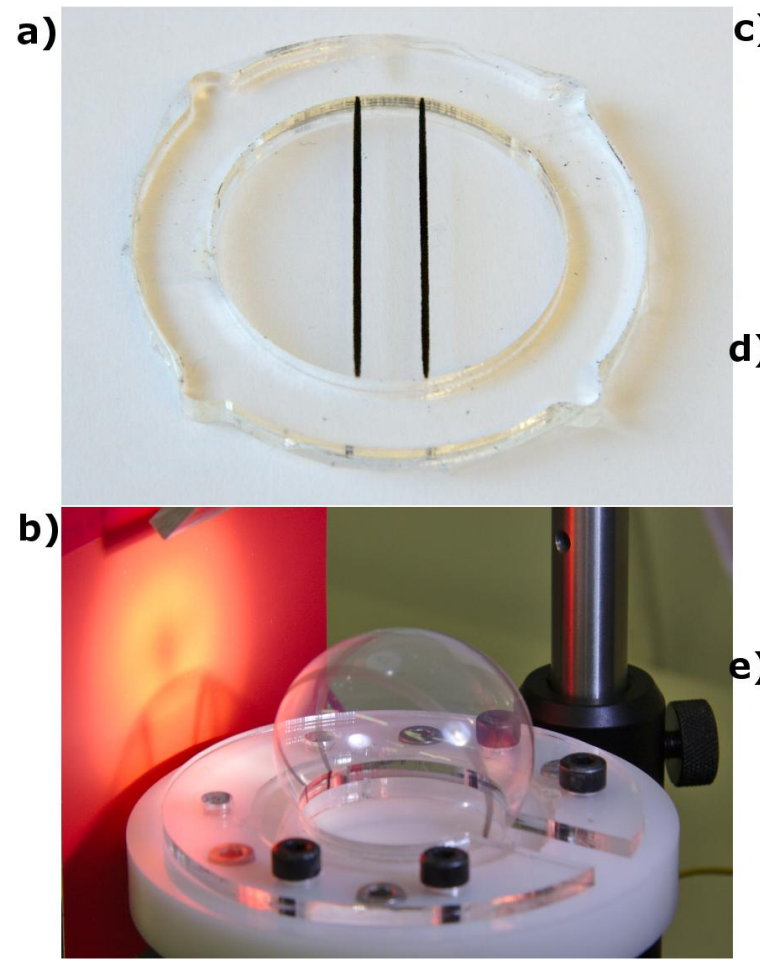

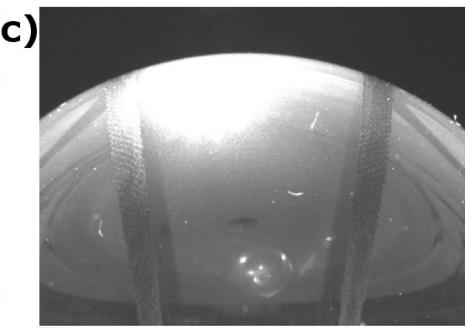

d)

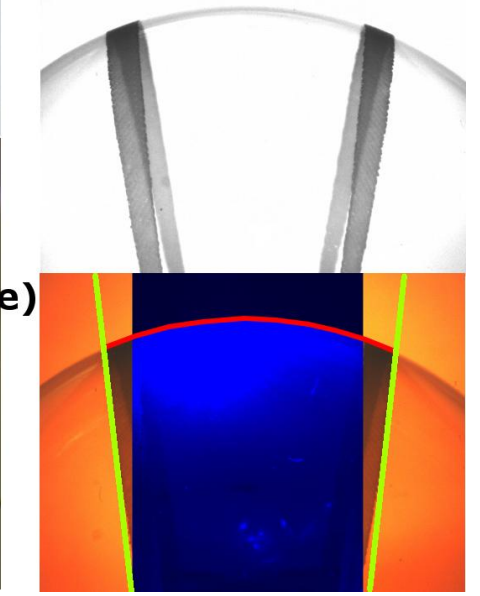

Figure 5. Equi-biaxial bubble tester. a) Membrane ready to be tested with two compliant black lined to delimit the region of interest at the center. b) Membrane inflated on the holder. c) Image with green illumination to highlight the curvature. d) Image with white illumination to highlight the delimiting lines. e) Processed image with the detected boundaries as overlay.

the front. For each pressure step, 2 pictures are taken. One with a green illumination coming from the top to highlight the dome outline, and one with a white light coming from the front to highlight the two black lines. A LabView interface is used to command the pressure source, the two light sources and the camera, as well as to analyse the image in order to find the radius of curvature of the bubble and the stretch, which is calculated by computing the length of the arc between the two printed lines (red overlay on figure 5 e).

A CF19 silicone membrane from the same batch as the samples used for the pull-test was characterized with the setup up to different stretch ratios (the pressure used for each step is given in parenthesis): 1.58 (20 mbar), 1.84 (22.5 mbar), 2.19 (26 mbar), 2.56 (30 mbar), and 3.17 (36 mbar). A sacrificial-layer-based fabrication process similar to the one described in section 2 was used to ensure that the membrane has seen no deformation before the test. It should be noted that the final stretch ratio cannot directly be controlled: the user chooses the pressure range to apply to the membrane, and it is only after the images are processed that the stretch value is known, hence the irregularly-spaced sequence. The upper stretching values of the uniaxial pull-test presented in section 2 where chosen according to table 2 to keep $I_{1}$ equal, which therefore also explains the spacing between the uni-axial tests. In addition, in order for the image analysis to work correctly, some parameters need to be set, such as camera exposure time, contrast and brightness, region of interest, threshold parameters, etc. before a measurement is performed. This involves inflating the membrane before the test, meaning that unlike for the uniaxial pull-test, the behaviour of the elastomer when it is stretched to a certain value for the first time (similar to what is shown on figure 3) cannot be captured by the measurement setup. Only the second and later inflations can be measured, leading to results similar to figure 4 for the uniaxial case.

Figure 6 shows the experimental equi-biaxial data obtained with the bubble tester. The red line represents a fit of the Gent model for the equi-biaxial case on the experimental data points, and the corresponding model parameters are given in table 3. Although the predicted behaviour from the uni-axial data gives an acceptable approximation (green line), it is far from perfect. The shear modulus obtained by uniaxial pull test is lower than in the equi-biaxial case, which, when applied to actuator will tend to underestimate the voltage required 

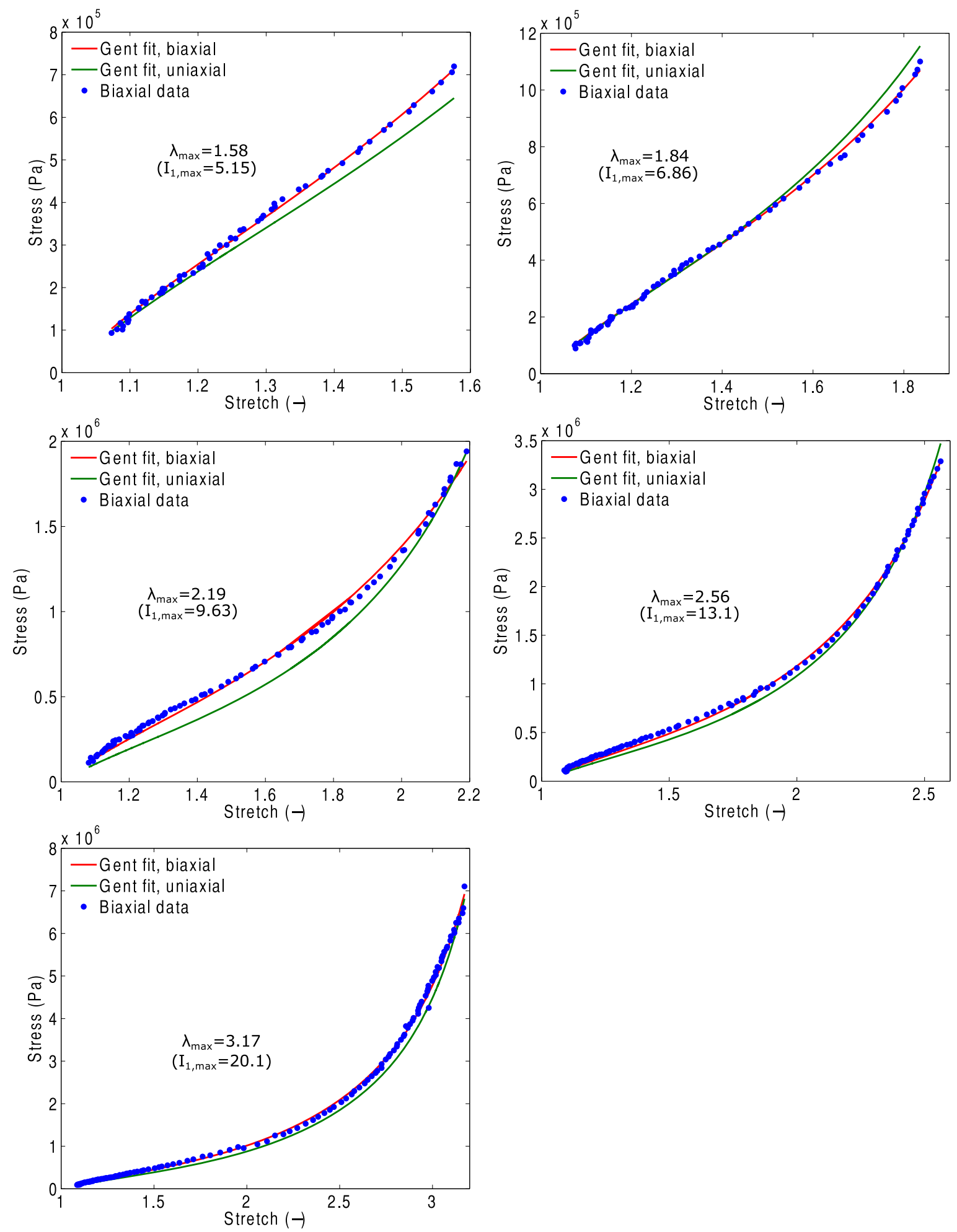

Figure 6. CF19 equi-biaxial stress-stretch curves for different maximal stretches obtained with the bubble tester. The blue dots represent the experimental data, the red line is a fit of the Gent model on the data, and the green line represents the Gent model calculated with the parameters obtained through the uniaxial tension test at identical maximal $I_{1}$, given in table 1 


\begin{tabular}{|l|c|c|c|c|c|}
\hline Maximal stretch & $\mathbf{1 . 5 8}$ & $\mathbf{1 . 8 4}$ & $\mathbf{2 . 1 9}$ & $\mathbf{2 . 5 6}$ & $\mathbf{3 . 1 7}$ \\
\hline$\mu(\mathrm{MPa})$ & 0.259 & 0.251 & 0.258 & 0.215 & 0.204 \\
\hline$J_{m}$ & 13.65 & 16.72 & 19.06 & 17.75 & 24.34 \\
\hline
\end{tabular}

Table 3. Values of the shear modulus $\mu$ and the $J_{m}$ parameter obtained by fitting the Gent model to experimental data points of the equi-biaxial bubble inflation tests.

to achieve a given strain. The figure also shows that the Gent model does not perfectly model the behaviour of the CF19 silicone. This is especially visible on the $\lambda_{\max }=2.19$ test: the model underestimate the stress at low stretches and underestimate it at higher stretches. This is also present on the two next tests at higher stretch, but less visible on the graph due to the higher $y$ scale. The impact of this effect will be discussed in the section 4 .

\section{STRETCH-VOLTAGE BEHAVIOUR OF DEAS}

The main motivation behind the accurate modelling of the stress-stretch curve in the field of DEAs is the ability to predict the behaviour of the actuator once the voltage is applied to the structure. In the case of the simple perfectly equi-biaxial actuator with free boundary conditions presented earlier (section 1), the relation between the in-plane expansion of the electrodes $\lambda_{e}$ and the applied voltage $V$ is given by: ${ }^{12}$

$$
V=\frac{z}{\lambda_{e}^{2} \sqrt{\epsilon}} \sqrt{\sigma_{b}\left(\lambda_{p} \lambda_{e}\right)-\sigma_{b}\left(\lambda_{p}\right) \lambda_{e}}
$$

Where $z$ is the thickness of the membrane after prestretch at $0 \mathrm{~V}, \epsilon$ is the electric permittivity of the dielectric, $\lambda_{p}$ is the prestretch value, and $\sigma_{b}$ is the biaxial stress-stretch function derived from the strain energy density function. The elastomer used as membrane for the actuator impacts the equation on different levels: 1) the equibiaxial stress-stretch behaviour as exposed above, 2) its permittivity $\epsilon$ assumed to be equal to $3 \times 8.85 \cdot 10^{-12} \mathrm{~F} / \mathrm{m}$ in the following calculations and 3) its dielectric breakdown strength which ultimately determines how far along the voltage-stretch curve the actuator can go before breakdown occurs.

Because of the stretch-induced softening, an actuator is not characterized by a single curve in the voltagestretch plane, but by many curves depending on the maximal stretch seen by the dielectric membrane (a better representation would be a plane in 3D space with $\lambda_{\max }$ as the third dimension. Figure 7 shows the predicted voltage-stretch behaviour for a $30 \mu \mathrm{m}$-thick CF19 silicone membrane (thickness after prestretch) for two different prestretch values $\lambda_{p}: 1.2$ (left), and $\sqrt{2}$ (right). The dashed black line represent the breakdown voltage for an estimated dielectric breakdown field of $150 \mathrm{~V} / \mu \mathrm{m}$. Any point on the right of this limit leads to the breakdown of the actuator. Because the figure shows the actuation stretch $\lambda_{e}$ and not the total stretch in the active region, the results are plotted from 1 to $\lambda_{e, \max }=\lambda_{\max } / \lambda_{p}$. The results show that the softening of the elastomer leads to drastically different behaviour of the device depending on the maximal deformation that the dielectric has sustained.

Looking at the case $\lambda_{p}=1.2$, the characteristic can shift from a behaviour without instability (curve monotonically increasing) for low maximal stretch to a behaviour showing a clear instability at larger stretching. A device which has not yet been activated can initially sustain an actuation voltage of $2100 \mathrm{~V}$, but repetitive actuation at that voltage will lead to an increasing strain and and to mechanical instability. It can be argued that the actuator will never show a behaviour shown by the curves $\lambda_{\max }=2.19-3.17$, as it will reach breakdown before reaching this strain levels. However the maximal strain seen by the actuator is not necessarily electrostatically induced. It can for example be applied during prestretch, by deforming the membrane to a higher value before coming back to 1.2, or unwillingly during fabrication, when the silicone membrane is peeled off from the casting substrate.

One additional important factor influencing the voltage-stretch behaviour is the method used to obtain the parameters for the hyperelastic model. Both uniaxial (table 1) and equi-biaxial (table 3) tests have been performed and have lead to slightly different values of the model parameters. Uniaxial tests are easier to perform, but the equi-biaxial results are expected to be more representative of the actuator behaviour, which is also in an equi-biaxial stretch state. Figure 8 shows the predicted voltage-stretch behaviour using Gent model parameters 

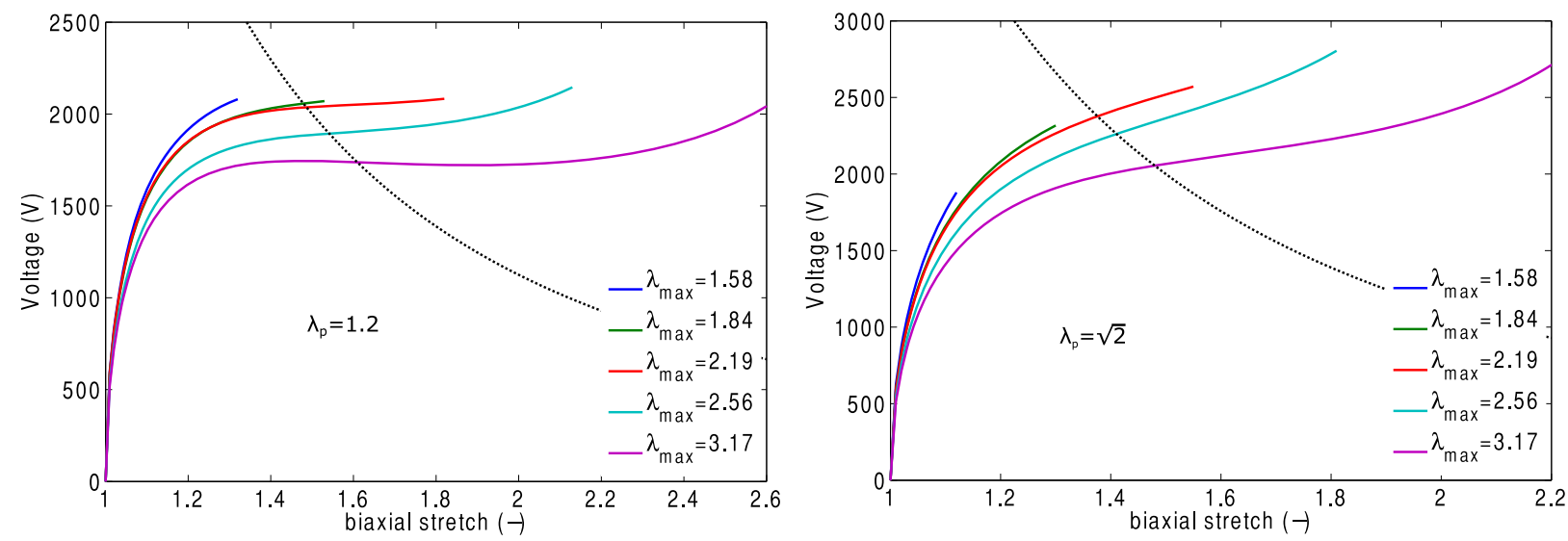

Figure 7. Predicted voltage-stretch behaviour for a $30 \mu \mathrm{m}$-thick CF19 silicone membrane (thickness after prestretch) for two different prestretch values: 1.2 (left), and $\sqrt{2}$ (right). The curves are calculated using the Gent model with the parameters obtained from the equi-biaxial stretching test (table 3). The dashed black lines represent the breakdown limit for an electric field of $150 \mathrm{~V} / \mu \mathrm{m}$. The $x$ axis of the graphs represents the actuation stretch (stretch seen on the electrodes) and not the total stretch of the membrane.

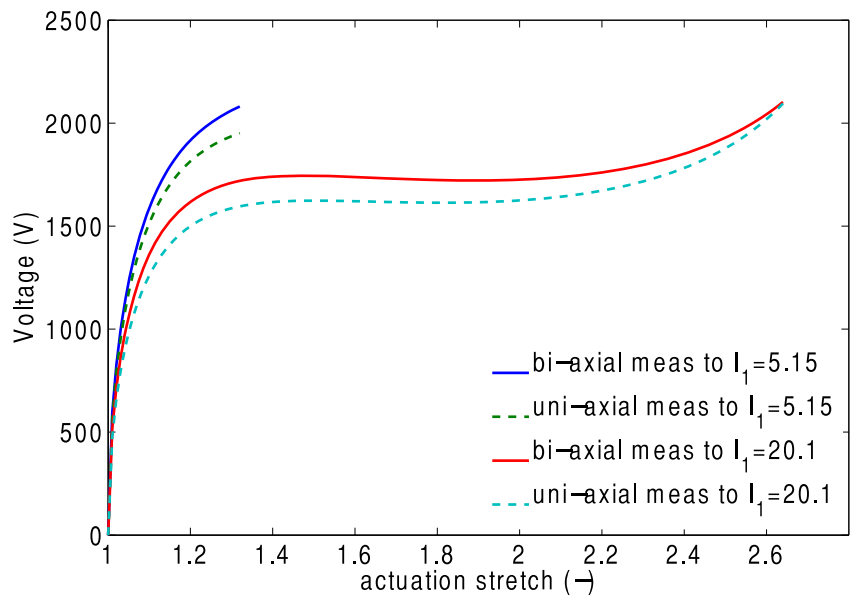

Figure 8. Voltage-stretch behaviour for $\lambda_{p}=1.2$ for the minimal and maximal studied stretching range, predicted with the Gent model fitted on both the uni-axial and equi-biaxial experimental tests.

obtained either from the uniaxial or equi-biaxial stretching test. Two extreme cases are represented: the smallest $I_{1}$ tested (5.15, equivalent to a maximal $\lambda_{b}$ of 1.58), and the largest $I_{1}$ (20.1, equivalent to a maximal $\lambda_{b}$ of 3.17). It shows that the smaller shear Modulus obtained with the uniaxial pull-test leads to an underestimation of the voltage required to obtain a given strain.

The Gent model does not provide a perfect description of the stress-stretch behaviour of the silicone CF19 (see figure 6). Consequently the voltage-stretch curves calculated using this model (figures 7 and 8) do not correspond exactly to the tested material. However, as the test actuator is used in a purely equi-biaxial condition,

\begin{tabular}{|c|c|c|c|c|c|}
\hline$\lambda_{\max }$ & $\mathbf{1 . 5 8}$ & $\mathbf{1 . 8 4}$ & $\mathbf{2 . 1 9}$ & $\mathbf{2 . 5 6}$ & $\mathbf{3 . 1 7}$ \\
\hline p0 (MPa) & -4.87 & -5.11 & -5.49 & 1.43 & 4.09 \\
\hline p1 (MPa) & 9.76 & 10.1 & 10.6 & -6.60 & -11.8 \\
\hline p2 (MPa) & -6.57 & -6.62 & -6.69 & 8.86 & 12.1 \\
\hline p3 (MPa) & 1.67 & 1.61 & 1.55 & -4.50 & -5.04 \\
\hline p4 (MPa) & 0 & 0 & 0 & 0.842 & 0.782 \\
\hline
\end{tabular}

Table 4. Polynomial coefficients $\sigma_{b}=\sum p n \cdot \lambda_{b}^{n}$ for the experimental equi-biaxial data at the different maximal stretch values 

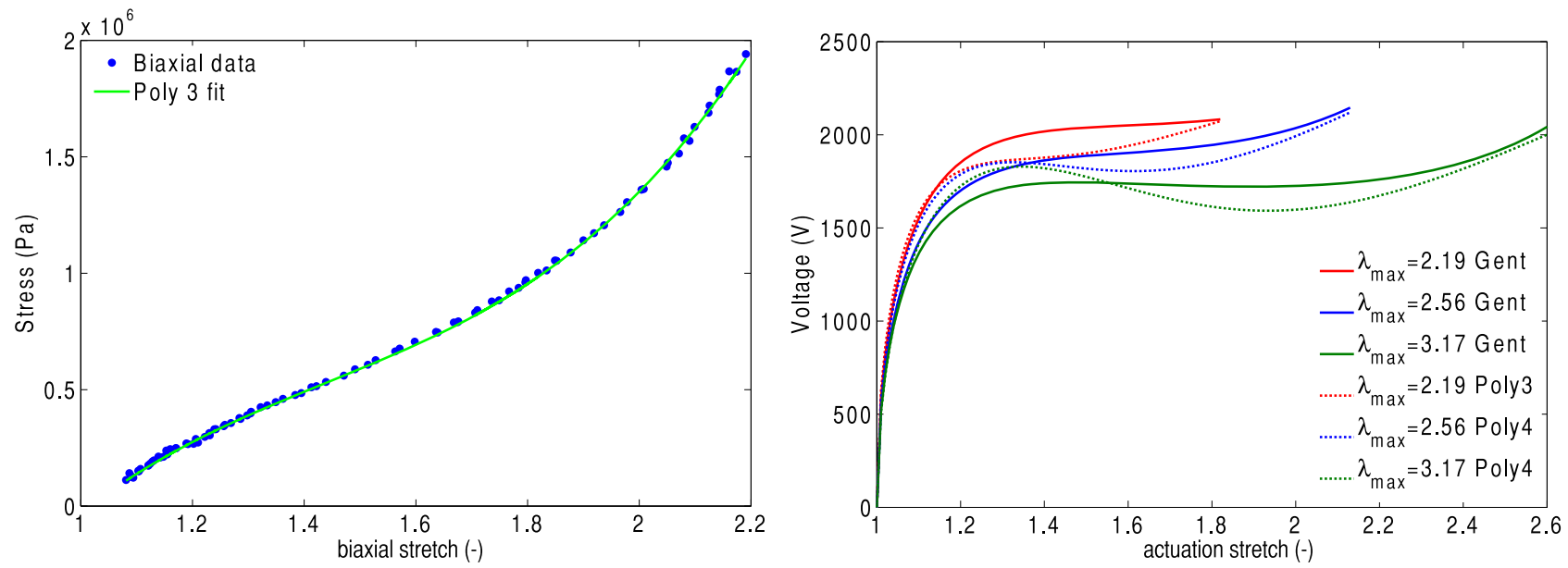

Figure 9. Left: fitting of the experimental equi-biaxial stretching test up to $I_{1, \max }=9.63\left(\lambda_{b, \max }=2.19\right)$ with a 3 rd order polynomial. Right: comparison of the voltage-stretch curve for $\lambda_{p}=1.2$ obtained from the equi-biaxial test, with the Gent model and with a polynomial fit of degree 3 or 4.

and the bubble tester gives us experimental equi-biaxial stress-stretch points, a strain energy density model is not necessary. The voltage equation (4) can be computed using the experimental data as $\sigma_{b}$. Better: to remove the noise and provide a way of interpolating between the discreet measurement points, a polynomial fit can be performed on the data. Excellent fitting is obtained with a 3rd order polynomial for the $I_{1, \max } 5.15,6.86$, and 9.63 (see figure 9 left for the $\lambda_{\max }=2.19$ case), and a 4th order polynomial was used with the two higher $I_{1, \max }$. The respective coefficients are given in table 4 . The voltage-stretch curve is then calculated using the polynomial approximation to model the stress instead of the hyperelastic Gent model. No noticeable difference is seen for the two lower stretch ranges $\left(I_{1, \max }=5.15\right.$ and $\left.I_{1, \max }=6.86\right)$ : the Gent model is a good representation of the stress-stretch data for this limited stretch region. For the 3 other ranges, an important difference is observed, and the result obtained using the Gent model and the polynomial approximation is given on figure 9 right. Because the Gent model underestimates the stress at low stretches, it predicts a too low actuation voltage in this range. In addition, as it overestimates the stress at higher stretch values, it also overestimates the voltage. Consequently the polynomial approximation (which we can consider as the best approximation as it fits the experimental stress-stretch data the best), shows a different behaviour, which can, in certain circumstances be drastically discrepant. For example, according to the Gent model, the curve up to $\lambda_{\max }=2.56$ does not present electromechanical instability, while it is clearly visible with the polynomial approximation.

\section{CONCLUSIONS}

In principle, modelling a simple free boundary conditions equi-biaxial ideal DEA should be as simple as applying an equation using a material model with parameters fitted by an experimental test such as an uniaxial pull-test. However, in the case of silicones (more specifically Nusil CF19-2186 and Dow Corning Sylgard 186 which we regularly use for our DEAs, but the same behaviour is expected to be observed to some extent on any silicone), the presence of a strong stretch-induced softening of the material makes modeling more difficult, as the deformation history of the membrane must be known. This also stresses the importance to avoid deforming the membrane during the fabrication process, as it can lead to unexpected results, such as anisotropic behaviour in case the membrane is released from its casting substrate by pulling unidirectionally on it.

The results also pinpoints the limitation of hyperelastic models for silicone elastomers. Although the Gent model is appealing because it can model the rapid stiffening of the material at a finite stretch values, experimental data show that it fails to accurately model the behaviour of the elastomer on an extended stretch range, and that parameters obtained by fitting on data in a particular condition (such as uniaxial) fail to accurately represent the material in another stretching condition (such as equi-biaxial). As shown with the results presented in this study, the predicted behaviour of a DEA will be different depending if the parameters of the hyperelastic model were fitted on uniaxial or biaxial data, or if a polynomial approximation was used. One of the parameters a user 
can expect to extract from DEA modelling can be for example the prestretch level that should be used in order to suppress electromechanical instability and obtain strain larger than $23 \%$ while not stiffening the actuator too much. However, we can see that this task is difficult, because for the exact same actuator and prestretching conditions, stable or unstable behaviour is predicted, depending on the set of parameters used to model the elastomer.

Because in many DEA applications the membrane is equi-biaxially prestretched, and during actuation the active area is also equi-biaxially stretched, it is therefore more accurate to characterize the stress-stretch behaviour of the dielectric membrane in an equi-biaxial configuration. We have built a bubble tester for this very purpose: the test setup inflates a thin elastomeric membrane, which is under an equi-biaxial stretch state at its center. From the applied pressure, the stretch and radius of curvature at the dome apex, the bi-axial stress-stretch characteristic can be measured. Hyperelastic models are useful when the testing conditions do not match the usage conditions (for example uni-axial test and equi-biaxial usage), as it allows to recalculate the stress-stretch curve for any situation. However, in the case of an equi-biaxial DEAs and stress data obtained with the bubble tester, using an hyperelastic model is not necessary, as the equi-biaxial stress behaviour is already known. Consequently, a simple polynomial approximation of the experimental data (of an order sufficiently high to accurately represent the data set) can lead to more realistic results. It is however common that the actuator is not in a simple equi-biaxial stress-state, for example when complex boundary conditions come into play (electrode near the border of the membrane), or if asymmetric prestretch is used. ${ }^{4}$ In that case, using a strain energy density function is mandatory, but the question remains on which parameters should be used for the model: we have seen that uniaxial and equi-biaxial tests lead to slightly different parameter values. Other models should be tested to investigate if they can more accurately model the behaviour of the silicone in different stretching conditions.

In addition, most of the published modelling study on DEAs, including this one, do not take into account the impact of the compliant electrodes placed on each side of the dielectric. There are many different kind of compliant electrodes used to make DEAs, mainly based on carbon or metal. ${ }^{13}$ None of them is perfectly compliant and their mechanical impact on the stiffness of the actuator sandwich should be taken into account if a precise modelling of DEAs is desired. Recently, Bozlar et al. have proposed a model based on hyperelastic

strain energy density functions to model a DEA with elastomeric electrodes that takes into account the strain energy stored into the electrodes. ${ }^{5}$

\section{ACKNOWLEDGMENTS}

This work has been funded by the Swiss National Science Foundation grant 200020-140394.

\section{REFERENCES}

1. Z. Suo, "Theory of dielectric elastomers," Acta Mechanica Solida Sinica 23(6), pp. 549-578, 2010.

2. S. Koh, T. Li, J. Zhou, X. Zhao, W. Hong, J. Zhu, and Z. Suo, "Mechanisms of large actuation strain in dielectric elastomers," Journal of Polymer Science, Part B: Polymer Physics 49(7), pp. 504-515, 2011.

3. C. Keplinger, T. Li, R. Baumgartner, Z. Suo, and S. Bauer, "Harnessing snap-through instability in soft dielectrics to achieve giant voltage-triggered deformation," Soft Matter 8, pp. 285-288, 2012.

4. S. Akbari, S. Rosset, and H. R. Shea, "Improved electromechanical behavior in castable dielectric elastomer actuators," Applied Physics Letters 102(7), p. 071906, 2013.

5. M. Bozlar, C. Punckt, S. Korkut, J. Zhu, C. Chiang Foo, Z. Suo, and I. A. Aksay, "Dielectric elastomer actuators with elastomeric electrodes," Applied Physics Letters 101(9), p. 091907, 2012.

6. T. Li, C. Keplinger, R. Baumgartner, S. Bauer, W. Yang, and Z. Suo, "Giant voltage-induced deformation in dielectric elastomers near the verge of snap-through instability," Journal of the Mechanics and Physics of Solids 61(2), pp. $611-628,2013$.

7. M. Kollosche, J. Zhu, Z. Suo, and G. Kofod, "Complex interplay of nonlinear processes in dielectric elastomers," Phys. Rev. E 85, p. 051801, May 2012.

8. L. Maffli, S. Rosset, and H. R. Shea, "Zipping dielectric elastomer actuators: characterization, design and modeling," Smart Materials and Structures 22(10), p. 104013, 2013. 
9. A. N. Gent, "A new constitutive relation for rubber," Rubber Chemistry and Technology 69, pp. 59-61, March 1996.

10. L. Mullins, "Softening of rubber by deformation," Rubber Chemistry and Technology 42(1), pp. 339-362, 1969.

11. N. Reuge, F. Schmidt, Y. Le Maoult, R. M., and F. Abbe, "Elastomer biaxial characterization using bubble inflation technique. i: Experimental investigations," Polymer Engineering and Science 41(3), pp. 522-531, 2001.

12. S. Rosset, P. Gebbers, B. M. O'Brien, and H. R. Shea, "The need for speed," in Proceedings of SPIE - The International Society for Optical Engineering, 8340, pp. 834004-834004-12, 2012.

13. S. Rosset and H. Shea, "Flexible and stretchable electrodes for dielectric elastomer actuators," Applied Physics A 110, pp. 281-307, 2013. 